EPJ Web of Conferences 81, 06003 (2014)

DOI: $10.1051 /$ epjconf/ 20148106003

(C) Owned by the authors, published by EDP Sciences, 2014

\title{
Energy Calibration for the Forward Detector at WASA-at-COSY
}

\author{
Kay Demmich ${ }^{1, a}$ \\ for the WASA-at-COSY Collaboration \\ ${ }^{1}$ Institut für Kernphysik, Westfälische Wilhelms-Universität Münster, Germany
}

\begin{abstract}
Studies on rare and forbidden decays of light mesons are one main aspect of the WASA-at-COSY physics program. In this context a large data set for $\eta$ mesons has been produced in the proton-proton scattering in order to investigate the decay properties of this meson. This high statistic measurement allows, e.g., for the search for the $C$-parity violating reaction $\eta \rightarrow \pi^{0}+e^{+}+e^{-}$. This article presents a new calibration method which allows for an improved separation of the physical background by means of the missing mass method.
\end{abstract}

\section{Introduction}

The WASA experiment at the COSY accelerator facility is dedicated to study the production and the decays of light mesons [1]. In order to investigate the decay channels of the $\eta$ meson a data set with very high statistics was taken in 2008, 2010, and 2012 in proton-proton collisions providing a sample with approx. $5 \times 10^{8}$ $\eta$ events. This large amount of data allows for the study on several decay modes of the $\eta$ meson, especially for rare and forbidden decays like $\eta \rightarrow \pi^{0}+e^{+}+e^{-}$. Since the $C$-parity allowed process involving two virtual photons, i.e. $\eta \rightarrow \pi^{0}+\gamma^{*}+\gamma^{*} \rightarrow \pi^{0}+e^{+}+e^{-}$, is expected to be strongly suppressed, the study on this decay gives the possiblity to search for $C$-parity violating decays via one virtual photon $\eta \rightarrow \pi^{0}+\gamma^{*} \rightarrow \pi^{0}+e^{+}+e^{-}$in combination with the vector meson dominance assumption (see Fig. 1). This particular

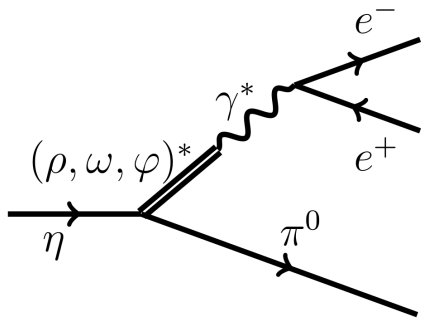

Figure 1. The decay $\eta \rightarrow \pi^{0}+$ $e^{+}+e^{-}$assuming a vector meson dominance model. reaction violates the $C$-parity according to:

$$
C=C_{\pi^{0}} \cdot C_{\gamma^{*}}=(+1) \cdot(-1)=-1 \neq+1=C_{\eta} .
$$

In addition, such studies allow for the search of a $Z$-like dark $U$ boson as intermediate state coupling to the lepton pair.

Not being observed yet, only an upper limit for the branching ratio of this decay assuming a phase space distribution for the ejectiles was found in former experiments and is quoted to be

$$
\mathrm{BR}\left(\eta \rightarrow \pi^{0}+e^{+}+e^{-}\right)<4 \times 10^{-5} \quad \text { C.L. }=90 \%[2] .
$$

\footnotetext{
a e-mail: Kay.Demmich@uni-muenster.de
} 
One analysis effort of the high statistic data obtained with WASA-at-COSY aims at lowering this upper limit as well as for constraining possible mass regions of a $Z$-like dark boson.

\section{Calibration}

Analyzes of rare and forbidden decays rely on an optimal detector resolution. To distinguish $\eta$ events and background events like direct multi pion productions the missing mass method is applied to the forward scattered protons. Hence, the high background reduction, which is necessary to analyze a forbidden decay, makes a careful calibration of the forward detector inevitable.

The kinetic energy of a forward scattered particle is calculated by its energy deposit $\Delta E$ in consecutive scintillator planes. This reconstruction relies on a calibration of the detector output ADC values and is done in two steps

$$
(A D C, \theta) \stackrel{f_{\mathrm{NU}}(\theta)}{\longmapsto} A^{\prime} \stackrel{f_{\mathrm{NL}}}{\longmapsto} \Delta E,
$$

where $\theta$ is the polar angle of the particle and $f_{\mathrm{NU}}$ and $f_{\mathrm{NL}}$ are two correction functions for the nonuniformity and the non-linearity effect respectively. The parameters of these functions have to be found in a calibration procedure.

In a first step, $f_{\mathrm{NU}}(\theta)$ can be determined by elastically scattered protons, which are minimum ionizing in good approximation due to their high energy. Their angular distribution of energy deposits $\frac{\mathrm{d} E}{\mathrm{~d} x}$ is expected to be flat. Any deviation from this constant behavior can be ascribed to a detector non-uniformity and the calibration function can be fitted to correct for this effect (see Fig. 2, left). The main reason for an angular dependency of the energy measerument is the geometrical design of the detector elements resulting in different path ways of the scintillation light to the photomultiplier.

In the second step, any small non-linearities in the detection system have to be corrected for. This effect results into an energy dependent correlation between the physical deposited energy loss $\Delta E$ and the measured (and non-uniformity corrected) value $A^{\prime}$. To correct for this non-linearity a calibration function $f_{\mathrm{NL}}$ is applied for which calibration parameters have to be determined. Protons populate characteristic regions in $\Delta E$-plots forming energy loss bands. The calibration is based on comparing these energy loss bands in real data and Monte Carlo simulations. Both data sets are visualized as two dimensional histograms (see Fig. 2, right). A calibration algorithm is developed which compares both
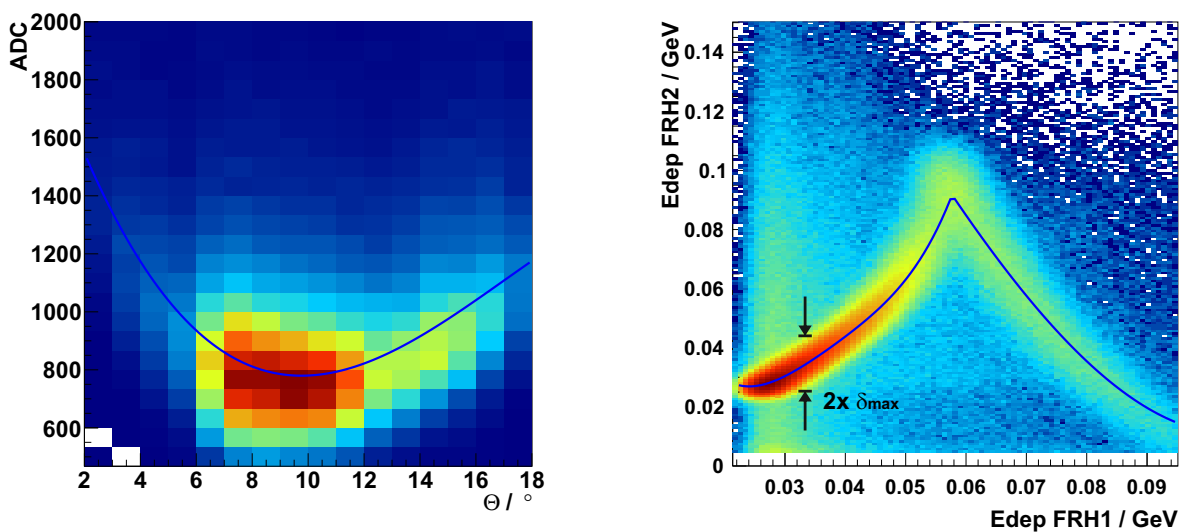

Figure 2. Left: angular distribution of elastically scattered protons and correction function $f_{\mathrm{NU}}$. Right: function $f_{\mathrm{MC}}$ fitted to a $\Delta E$-plot of Monte Carlo data. 
data sets and varies the calibration parameters to find a minimum discrepancy between the data sets. This algorithm first fits a function $f_{\mathrm{MC}}$ to the Monte Carlo data set using an estimator based on least squares and adjusted for fitting $2 \mathrm{~d}$ histograms afflicted with background:

$$
\chi^{2}=\sum_{\text {bins }} w_{i} \cdot \min \left(\delta_{i}^{2}, \delta_{\max }^{2}\right)
$$

where $w_{i}$ is the bin content of the $i$-th bin and $\delta_{i}$ represents the vertical distance between the bin and the fitting function. $\delta_{\max }$ is a constant that limits the influence of uncorrelated background to the algorithm. Minimizing this $\chi^{2}$ value fits the function $f_{\mathrm{MC}}$ to the simulated distribution. Subsequently using the same algorithm the calibration parameters are varied to match the real data to $f_{\mathrm{MC}}$. In this way, a consensus of real and Monte Carlo data can be achieved and the calibration is completed.

Fig. 2 shows exemplarily the $\Delta E$-plot combining the first and second scintillator layer. The complete energy loss detector consists of five layers resulting in additional calibration parameters and $\Delta E$-plots. Thus, the calibration software performs the described algorithm simultaneously to all five layers and all possible layer combinations.

\section{Summary and outlook}

A new calibration algorithm allows for the fast calibration of the WASA forward detector using a new fitting approach which is able to fit real data present as a two dimensional histogram and afflicted with background to Monte Carlo data which are likewise present as an histogram. This innovation will improve the detector resolution and will help to lower the existing limit for the forbidden decay $\eta \rightarrow \pi^{0}+e^{+}+e^{-}$as well as improve other decay analyzes.

\section{Acknowledgements}

The support from COSY-FFE grants is kindly acknowledged.

\section{References}

[1] H.-H. Adam et al. (WASA-at-COSY Collaboration), arXiv:nucl-ex/0411038

[2] K.A. Olive et al. (Particle Data Group), Chin. Phys. C, 38, 090001 (2014) 